\title{
A Guide to the Use of the Modified Reflectometer Technique of VSWR Measurement *
}

\author{
Wilbur J. Anson
}

(February 10, 1961)

\begin{abstract}
The theory of the modified reflectometer technique of measuring VSWR at microwave frequencies is discussed briefly, the operational procedure is outlined, and selected results are given of an unpublished error analysis. Much of the theory and procedure has been published in isolated papers. This paper unifies those details essential to the use of this technique and includes procedural suggestions that have grown out of extensive experience with the technique. The error analysis provides the means to evaluate the accuracy of any particular measurement made with this system.
\end{abstract}

\section{Introduction}

The modified reflectometer technique [Beatty and Kerns, 1958; Beatty, 1959; Engen and Beatty, 1959] recently developed at the Boulder Laboratory of the National Bureau of Standards is a method of making accurate VSWR measurements in rectangular waveguide. This technique is capable of measuring low VSWR's (VSWR $<2$ ) to accuracies of 1 percent quite easily. Accuracies of 0.1 percent in VSWR are obtainable with extra care in the adjustments of the modified reflectometer.

The purpose of this paper is to provide operating knowledge of the modified reflectometer technique. Much of the material presented herein has been published elsewhere but this paper gathers in one place those details essential to the use of this technique. The paper also offers suggestions on procedure which have grown out of extensive experience with this technique. Also it provides selected results of an error analysis which are important in evaluating the accuracy of any particular measurement made with this system.

\section{An Outline of the Theory}

The modified reflectometer measures the magnitude of the reflection coefficient of a waveguide termination at microwave frequencies. This method utilizes a directional coupler oriented to couple to the reflected wave with appropriately placed tuners adjusted so that the amplitude of the voltage wave from the side arm is directly proportional to the magnitude of the reflection coefficient.

According to references 1 and 2, the scattering equations for a three arm junction representing the combination of a directional coupler and two tuners (fig. 1) are

$$
\begin{aligned}
& b_{1}=S_{11} a_{1}+S_{12} a_{2}+S_{13} a_{3} \\
& b_{2}=S_{21} a_{1}+S_{22} a_{2}+S_{23} a_{3} \\
& b_{3}=S_{31} a_{1}+S_{32} a_{2}+S_{33} a_{3},
\end{aligned}
$$

where $a_{1}=b_{g}+b_{1} \Gamma_{G}, a_{2}=b_{2} \Gamma_{L}$, and $a_{3}=b_{3} \Gamma_{D}$. These *Contribution from the Radio Standards Laboratory, National Bureau of Standards, Boulder, Colo. may be solved for the response in the form

$$
\begin{gathered}
b_{3}=b_{g} k \frac{\left(1+K \Gamma_{L}\right)}{\left(1-\Gamma_{2 i} \Gamma_{L}\right)}, \\
k=\frac{S_{31}}{\left|\begin{array}{ll}
\left(1-S_{11} \Gamma_{G}\right) & S_{13} \Gamma_{D} \\
S_{31} \Gamma_{G} & \left(1-S_{33} \Gamma_{D}\right)
\end{array}\right|},
\end{gathered}
$$

and

$$
K=\frac{\left|\begin{array}{ll}
S_{21} & S_{22} \\
S_{31} & S_{32}
\end{array}\right|}{S_{31}},
$$

$$
\Gamma_{2 i}=\frac{\left|\begin{array}{ccc}
-\left(1-S_{11} \Gamma_{G}\right) & S_{12} & S_{13} \Gamma_{D} \\
S_{21} \Gamma_{G} & S_{22} & S_{23} \Gamma_{D} \\
S_{31} \Gamma_{G} & S_{32} & -\left(1-S_{33} \Gamma_{D}\right)
\end{array}\right|}{\left|\begin{array}{cc}
\left(1-S_{11} \Gamma_{G}\right) & S_{13} \Gamma_{D} \\
S_{31} \Gamma_{G} & \left(1-S_{33} \Gamma_{D}\right)
\end{array}\right|} .
$$

Rearranged slightly, the equation becomes

$$
b_{3}=\frac{b_{g}}{\left|\begin{array}{cc}
\left(1-S_{11} \Gamma_{G}\right) & S_{13} \Gamma_{D} \\
S_{31} \Gamma_{G} & \left(1-S_{33} \Gamma_{D}\right)
\end{array}\right|} \frac{S_{31}+\left|\begin{array}{ll}
S_{21} & S_{22} \\
S_{31} & S_{32}
\end{array}\right| \Gamma_{L}}{1-\Gamma_{2 i} \Gamma_{L}} .
$$

Now let $S_{31}=0$ and $\Gamma_{2 i}=0$, then

$$
b_{3}=\frac{b_{g} S_{21} S_{32} \Gamma_{L}}{\left(1-S_{11} \Gamma_{G}\right)\left(1-S_{33} \Gamma_{D}\right)} .
$$

If $b_{g}, \Gamma_{G}$, and $\Gamma_{D}$ are constant, then

$$
b_{3}=C_{(\text {constant })} \Gamma_{L} .
$$

For a detector not phase sensitive

$$
\left|b_{3}\right|=\left|C \Gamma_{L}\right| .
$$

Note that this last relationship is the desired response mentioned just before (1), i.e., the amplitude of the voltage wave from the sidearm is directly proportional to the magnitude of the reflection coefficient. 
Therefore, let $\Gamma_{L}=\Gamma_{u}$ (the unknown) so that $\left|b_{3}\right|_{u}=\left|C \Gamma_{u}\right|$. Then let $\Gamma_{L}=\Gamma_{s}$ (a known quantity) so that $\left|b_{3}\right|_{s}=\left|C \Gamma_{s}\right|$. Now form the ratio

$$
\frac{\left|b_{3}\right|_{u}}{\left|b_{3}\right|_{s}}=\frac{\left|\Gamma_{u}\right|}{\left|\Gamma_{s}\right|}
$$

Thus, given the value of $\left|\Gamma_{s}\right|$, and measuring the ratio of the sidearm outputs $\frac{\left|b_{3}\right|_{u}}{\left|b_{3}\right|_{s}}$, the value of $\left|\Gamma_{u}\right|$ may be calculated.

\section{Procedure}

Any practical use of the modified reflectometer technique depends upon an understanding of the physical conditions imposed by the theory.

For $\left|b_{3}\right|$ to conform to (8) certain quantities as represented in (7) must remain constant. It requires a stable source (constant $b_{g}$ ) well isolated from load changes. It also requires apparent generator and detector impedances that are unchanging (constant $\Gamma_{G}$ and $\Gamma_{D}$ )-requirements which can be essentially fulfilled by isolators placed at terminal planes $T_{1}$ and $T_{3}$ in figures 1 and 2 .

The remaining quantities of (8), namely $S_{21}$, $S_{32}, S_{11}$, and $S_{33}$, are constants of the 3-arm junction and therefore remain unchanging after adjustments are made for $S_{31}=0$ and $\Gamma_{2 i}=0$.

\subsection{Adjustment of $S_{31}=0$ and $\Gamma_{2 i}=0$}

For a directional coupler with the arms labeled as in figure 2 the statement that $S_{31}=0$ is equivalent to saying that the coupler has infinite directivity since, in terms of scattering coefficients, directivity $\equiv 20 \log _{10}\left|\frac{S_{32}}{S_{31}}\right|$.

Infinite directivity can be approached by adjusting tuner $\mathrm{A}$ for no variation in the output $\left|b_{3}\right|$ when sliding a load of small reflection $\left(\left|\Gamma_{L}\right|<0.005\right)$ in the uniform waveguide.

After making this adjustment, the condition $\Gamma_{2 i}=0$ can be approached by adjusting tuner B for no variation in the output $\left|o_{3}\right|$ when sliding a load of large reflection (usually a shorting plunger) in the uniform waveguide.

This adjustment sequence may need to be repeated several times since the two adjustments are not

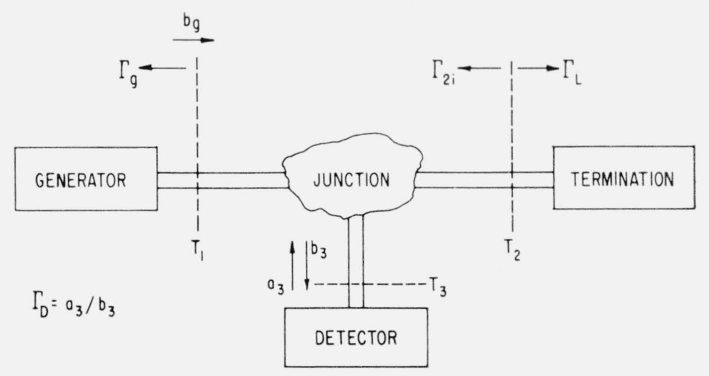

FIGURE 1. Three arm junction representing the modified reflectometer. completely independent. However, if the reflection from the sliding load used in the directivity adjust. ment is small enough, then the two adjustments are more nearly independent and one adjustment sequence will usually be enough.

Note: It is seldom necessary for the variation to be completely zero. The variation allowable while the loads are slid in each adjustment depends upon the desired accuracy of the measurement to be made. This allowable variation is discussed in the error analysis and is displayed in figures 5 , 6,7 , and 8 .

\subsection{An Alternate Adjustment Procedure for $S_{31}=0$ and $\Gamma_{2 i}=0$}

Consider the definition for directivity in conjunction with (6) and observe that $\left|b_{3}\right|=0$ when $\Gamma_{L}=0$ if the directivity is infinite. Therefore the adjustment could be made by attaching a flat load $\left(\Gamma_{L}=0\right)$ and adjusting tuner $\mathrm{A}$ for $\left|\boldsymbol{o}_{3}\right|=0$. But there is no commercial termination flat enough for the accuracy of adjustment often needed and therefore an adjustable sliding termination such as the one described by R. W. Beatty [1957] must be adjusted for $\Gamma_{L}=0$.

The two adjustments for $S_{31}=0$ and $\Gamma_{L}=0$, using an adjustable sliding termination, are carried out alternately. The adjustable termination is slid and adjusted so that the variations in $\left|b_{3}\right|$ are reduced and then tuner $\mathrm{A}$ is adjusted so that the average level of the variation is lowered. When no variation in $\left|b_{3}\right|$ occurs as the adjustable termination is slid, and when $\left|b_{3}\right|$ is zero, then $S_{31}=0$ and $\Gamma_{L}=0$. Note that after adjustment of tuner $A$ the variations will be greater than before. The greater variation results from increased sensitivity as infinite directivity is approached with the adjustments of tuner A.

Adjustments for $\Gamma_{2 i}=0$ can be made after making the previous adjustment for infinite directivity. The symbol $\Gamma_{2 i}$ in (2), besides representing a group of symbols as noted in (5), actually represents the reflection coefficient "looking in" arm 2 of the junction as indicated in figure 2. Therefore the condition $\Gamma_{2 i}=0$ is equivalent to saying that, at the terminal plane $T_{2}$, this input reflection coefficient is zero.

In figure 3 a scheme is illustrated for recognizing this matched condition. Power is coupled out of the main line to supply an auxiliary directional coupler

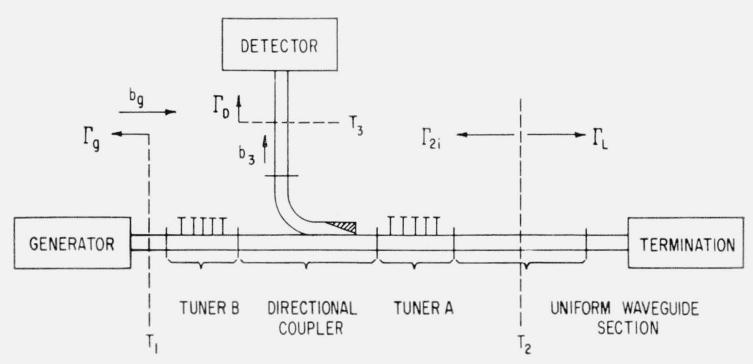

Figure 2. Diagram of modified reflectometer. 


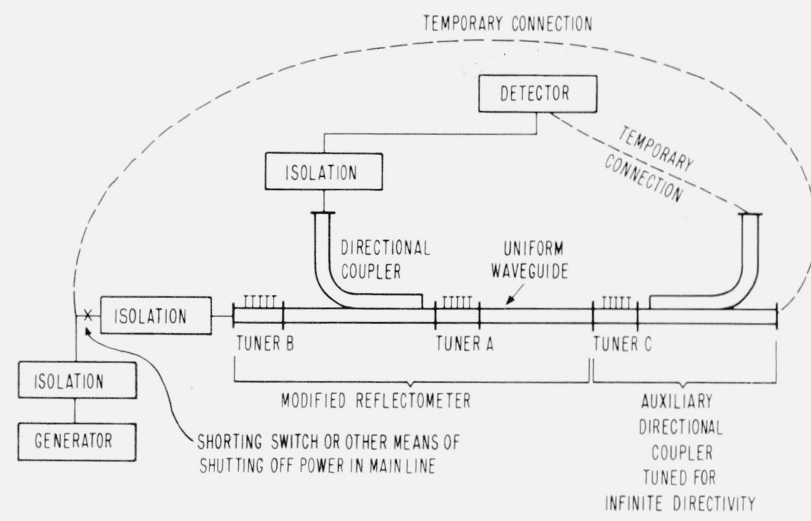

Figure 3. Equipment arrangement for adjusting $\Gamma_{2 i}=0$.

tuned for infinite directivity. This coupler is temporarily attached to the uniform waveguide with power shut off in the main line supplying the modified reflectometer. Tuner B is then adjusted for no output from the sidearm of the auxiliary coupler. The condition $\Gamma_{2 i}=0$ is thereby fulfilled.

There must, of course, be enough isolation on the generator side of the modified reflectometer to maintain essentially the same value of $\Gamma_{2 i}$ during both this matching procedure and the normal operation of the reflectometer. Also, there must be enough isolation between the generator and whatever means is used for shutting off the main line power to insure no possibility of frequency pulling.

The auxiliary directional coupler can be tuned for infinite directivity by utilizing the flat load previously discussed. This flat load is attached temporarily to the auxiliary coupler and tuner $\mathrm{C}$ adjusted for no output from the sidearm of the auxiliary coupler.

This adjustment sequence may also need to be repeated several times since the two adjustments are not completely independent. However, if the adjustment for infinite directivity is carefully made, one adjustment sequence will be enough.

Although this alternate procedure is sometimes easier to use, it does not provide sufficient information to evaluate the maximum errors due to imperfect adjustments of the tuners. Therefore, the adequacy of the adjustments must be checked by sliding the small and large reflection loads in the uniform waveguide and by observing whether the variations in $\left|b_{3}\right|$ are within the allowable limits for the measurement accuracy needed.

If the variations are not within the allowable limits it is important that the final adjustments be made with the small and large reflection sliding loads in the uniform waveguide. Otherwise, reflections from the waveguide joints will detune the infinite directivity adjustment.

\subsection{Another Alternate Adjustment Procedure for $S_{31}=0$}

By adding an auxiliary channel as illustrated in figure 4, the adjustment for infinite directivity $\left(S_{31}=0\right)$ is made considerably easier and faster [Beatty, Engen, and Anson, 1960]. This auxiliary

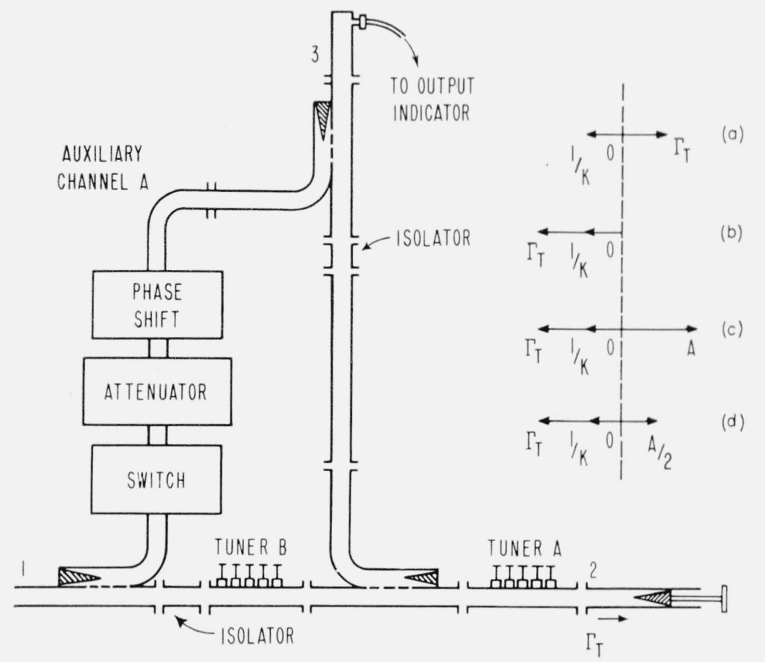

Figure 4. Auxiliary channel technique.

channel permits introduction of a signal of the proper phase and magnitude to null the signal component indicative of finite directivity.

From (2) the response from the sidearm is

$$
\left|b_{3}\right|=\left|b_{g} K\right|\left|\frac{1+K \Gamma_{L}}{1-\Gamma_{2 i} \Gamma_{L}}\right| .
$$

If $\left|\Gamma_{2 i}\right|$ is small, i.e., $\left|\Gamma_{2 i}\right|<0.01$, then a very good approximation is

$$
\left|b_{3}\right|=\left|b_{g} k K\right| \cdot\left|\frac{1}{K}+\Gamma_{L}\right| .
$$

The following description of the procedure is best understood by referring to (11) and the vector diagrams in figure 4.

1. With the shorting switch in the auxiliary arm closed, arbitrarily position the small reflection load in the uniform waveguide and adjust tuner $A$ for $b_{3}=0$. This corresponds to step (a) in figure 4 wherein $1 / K$ is oppositely directed and equal in magnitude to $\left|\Gamma_{L}\right|$.

2 . Slide the load for maximum $\left|b_{3}\right|$. This corresponds to step (b) in figure 4 wherein $\Gamma_{L}$ and $1 / K$ are in phase.

3. Open the waveguide switch in auxiliary channel $A$ and adjust the phase shifter and attenuator in that channel for $\left|b_{3}\right|=0$ again. This corresponds to step (c) in figure 4 which illustrates a signal A having been introduced to null $\left|\frac{1}{K}\right|+\left|\Gamma_{L}\right|$.

4. Introduce $6.02 \mathrm{db}$ of attenuation into the auxiliary arm to reduce the amplitude of signal A by one-half as illustrated in step (d) in figure 4. This $\mathrm{A} / 2$ cancels the $1 / K$ term in (11) and results in infinite directivity. Recall that $\left|\Gamma_{2 i}\right|$ is assumed small so that (11) is a good approximation.

The directivity obtained by this procedure can be checked by sliding the low reflection load and observing the variations in $\left|b_{3}\right|$ as noted in section 3.1. If the directivity obtained thusly is not high enough, then trial and error adjustments using the phase 
shifter and the attenuator in the auxiliary arm or tuner A will allow closer adjustment. However, adjustments using the components of the auxiliary arm are in general easier because these components usually present better resolution than tuner A.

To avoid possible interaction between the two channels, two isolators should be included as shown in figure 4. Other tuning [Engen, 1960] could be employed to reduce interaction but these two isolators should suffice.

\subsection{Measurement of $\left|\Gamma_{u}\right|$}

Certain details vary from system to system and from operator to operator depending upon personal preferences, equipment available and the accuracies needed. Therefore, no attempt has been made to write this guide for a specific arrangement of equipment.

However, regardless of the arrangement of the apparatus in the system, the ratio $\left|\frac{\Gamma_{u}}{\Gamma_{s}}\right|$ can be conveniently measured by some adaptation of the following sequence:

A. Adjust $S_{31}=0$ and $\Gamma_{2 i}=0$ by some combination of methods as previously outlined.

B. Check the adequacy of the adjustments to insure the achievement of the measurement accuracy required.

C. Attach the microwave termination of unknown reflection coefficient $\Gamma_{u}$ to the uniform wavegurde.

$D$. Set $\left|b_{3}\right|$ at some convenient reference level on some suitable detector and note the setting of a calibrated attenuator. This attenuator, of course, must be properly matched [Beatty, 1954; Schafer and Rumfelt, 1959] to measure accurately the voltage ratios at the detector.

E. Replace the unknown with some standard termination whose reflection coefficient is accurately known.

F. Return $\left|b_{3}\right|$ to the reference level by adjustment of the calibrated attenuator mentioned in step (D).

G. Record the difference in settings of the calibrated attenuator between step (D) and step (F). Calculate the value of $\left|\Gamma_{u}\right|$ from this difference, i.e., $\mathrm{R}=20 \log _{10}\left|\frac{\Gamma_{s}}{\Gamma_{u}}\right|$ where $R$ is the difference in decibels. This calculation can be aided by reference to a table [Beatty and Anson, 1960] of reflection coefficient versus return loss.

\section{The Error in the Measurement of $\left|\Gamma_{u}\right|$ and VSWR Due to $S_{31} \neq 0$ and $\Gamma_{2 i} \neq 0$}

As noted previously, the variation in $\left|b_{3}\right|$ allowable while the loads are slid in each adjustment depends upon the desired accuracy of the measurement made. This allowable variation can be computed from the following equations or taken from the graphs included for certain measurement situations. It is recommended that the adjustments be checked by this method both before and after measurements are made as an indication of changes created by accidental bumps or frequency drift.

\subsection{The Error Due to $S_{31} \neq 0$}

It can be shown that the maximum fractional error (assuming the worst phase combination) in the measurement of $\left|\Gamma_{u}\right|$, where $S_{31} \neq 0$ but all other conditions are met, is given by the expression

$$
\frac{d\left|\Gamma_{u}\right|}{\left|\Gamma_{u}\right|} \leq \frac{1}{\mid K}\left(\frac{\left|\Gamma_{s}\right|+\left|\Gamma_{u}\right|}{\left|\Gamma_{u}\right|-\left|\frac{\Gamma_{u} \mid}{K}\right|}\right)
$$

where $\Gamma_{s} \equiv$ reflection coefficient of the standard termination,

$\Gamma_{u} \equiv$ reflection coefficient of the unknown termination,

and

$$
|K|=\frac{10^{R_{1} / 20}+1}{\left(10^{R_{1} / 20}-1\right)\left|\Gamma_{L}\right|},
$$

in which $R_{1}$ is the db variation in $\left|b_{3}\right|$ as the small re flection load $\Gamma_{L}$ is slid in the uniform waveguide.

The corresponding maximum error in the measurement of VSWR, when $S_{31} \neq 0$, is obtained from (12) applying the following relation:

$$
\frac{d \sigma_{u}}{\sigma_{u}}=\frac{2\left|\Gamma_{u}\right|}{1-\left|\Gamma_{u}\right|^{2}} \frac{d\left|\Gamma_{u}\right|}{\left|\Gamma_{u}\right|} \text { where } \sigma_{u} \equiv \text { VSWR. }
$$

\subsection{The Error Due to $\Gamma_{2 i} \neq 0$}

The maximum fractional error in the measurement of $\left|\Gamma_{u}\right|$, when $\Gamma_{2 i}=0$ but all other conditions are met, is given by the expression

where

$$
\frac{d\left|\Gamma_{u}\right|}{\left|\Gamma_{u}\right|} \leq \frac{\left(\left|\Gamma_{u}\right|+\left|\Gamma_{s}\right|\right)\left|\Gamma_{2 i}\right|}{1-\Gamma_{2 i} \Gamma_{u}}
$$

$\Gamma_{s} \equiv$ reflection coefficient of the standard termination, $\Gamma_{u} \equiv$ reflection coefficient of the unknown termination, and $\left|\Gamma_{2 i}\right|$ is obtained from

$$
R_{2}=20 \log _{10} \frac{1+\left|\Gamma_{2 i} \Gamma_{L}\right|}{1-\left|\Gamma_{2 i} \Gamma_{L}\right|}
$$

in which $R_{2}$ is the db variation in $\left|b_{3}\right|$ observed as a large reflection load (usually a shorting plunger whose $\left|\Gamma_{L}\right| \cong 1$ ).

The corresponding maximum error in the measurement of VSWR, when $\Gamma_{2 i} \neq 0$, is obtained from (14) by applying (13).

\subsection{The Error Due to Both $S_{31} \neq 0$ and $\Gamma_{2 i} \neq 0$}

Seldom, if ever, will $S_{31}$ or $\Gamma_{2 i}$ be adjusted perfectly. However, it can be shown that the maximum error due to both $S_{31} \neq 0$ and $\Gamma_{2 i} \neq 0$ simultaneously is the sum of the individual maximum errors. Therefore, these individual errors may be obtained from (12) through (14), or from figures 5 through 8 , and added to form the maximum error of the measurement due to $S_{31} \neq 0$ and $\Gamma_{2 i} \neq 0$. 


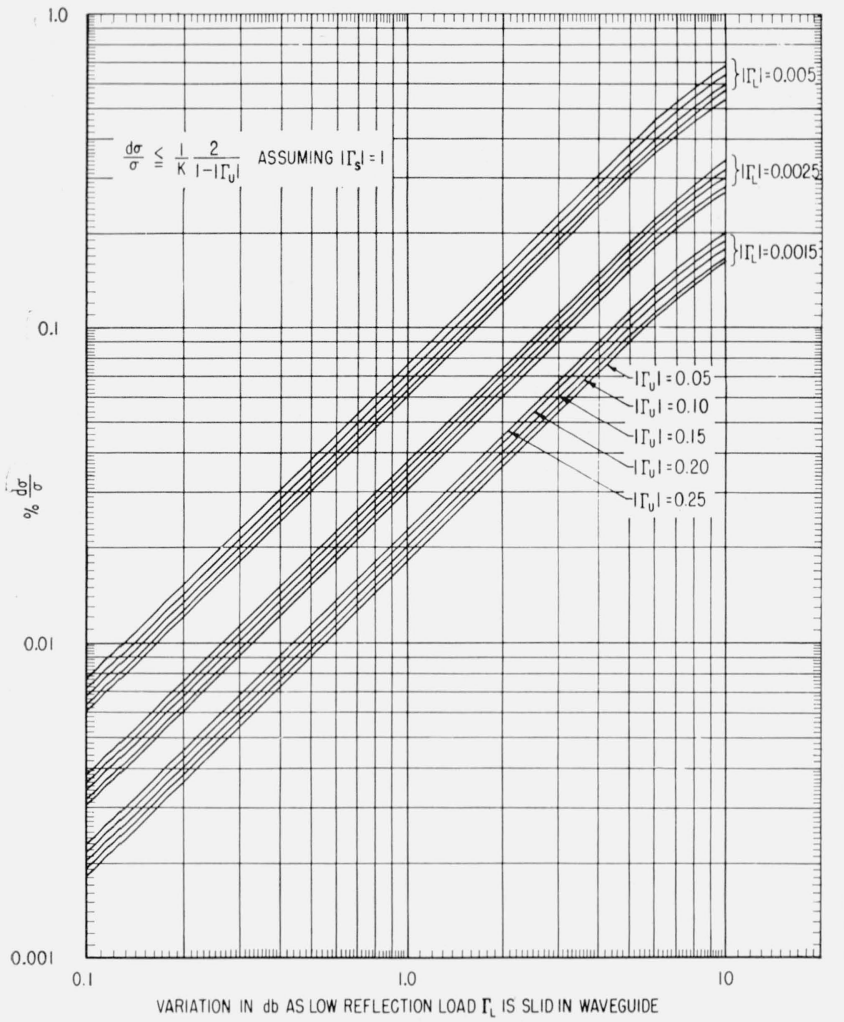

FIGURE 5. The maximum error in measuring VSWR by the modified reflectometer technique due to $S_{31} \neq 0$ and assuming $\Gamma_{2 i}=0$.

Example: Suppose there is $0.5 \mathrm{db}$ variation in $\left|b_{3}\right|$ as a small reflection load $\left|\Gamma_{L}\right|<0.005$ is slid in the uniform waveguide and there is a $0.02 \mathrm{db}$ variation as a short $\left(\left|\Gamma_{s}\right| \approx 1\right)$ is slid. What is the total limit of error in the measurement of the VSWR of a termination whose reflection coefficient is $\left|\Gamma_{u}\right| \approx 0.2$ ? From figure 5 the maximum error in measuring VSWR due to $S_{31} \neq 0$ is 0.036 percent; from figure 6 the maximum error due to $\Gamma_{2 i} \neq 0$ is 0.058 percent. So the maximum error (due to both $S_{31} \neq 0$ and $\Gamma_{2 i} \neq 0$ ) in measuring the VSWR of the termination is 0.094 percent.

\section{Procedural Suggestions}

1. Adjustment of the tuners for no variation in the sidearm response $\left|b_{3}\right|$ is easier if some systematic approach is used. One such approach is the follow. ing:

A. Slide the load for either a maximum or minimum response. In this example assume that it was slid for maximum.

B. Adjust a stub of the tuner in the direction which lowers the detector response. (Although this example uses a multiple-stub tuner the same procedure is usually adaptable to other types of tuners.)

C. Slide the load for a minimum.

D. Adjust the same stub in the same direction as before. If the response increases, i.e., moves toward the average of the variation, then further adjustment of that tuning stub using steps (A)

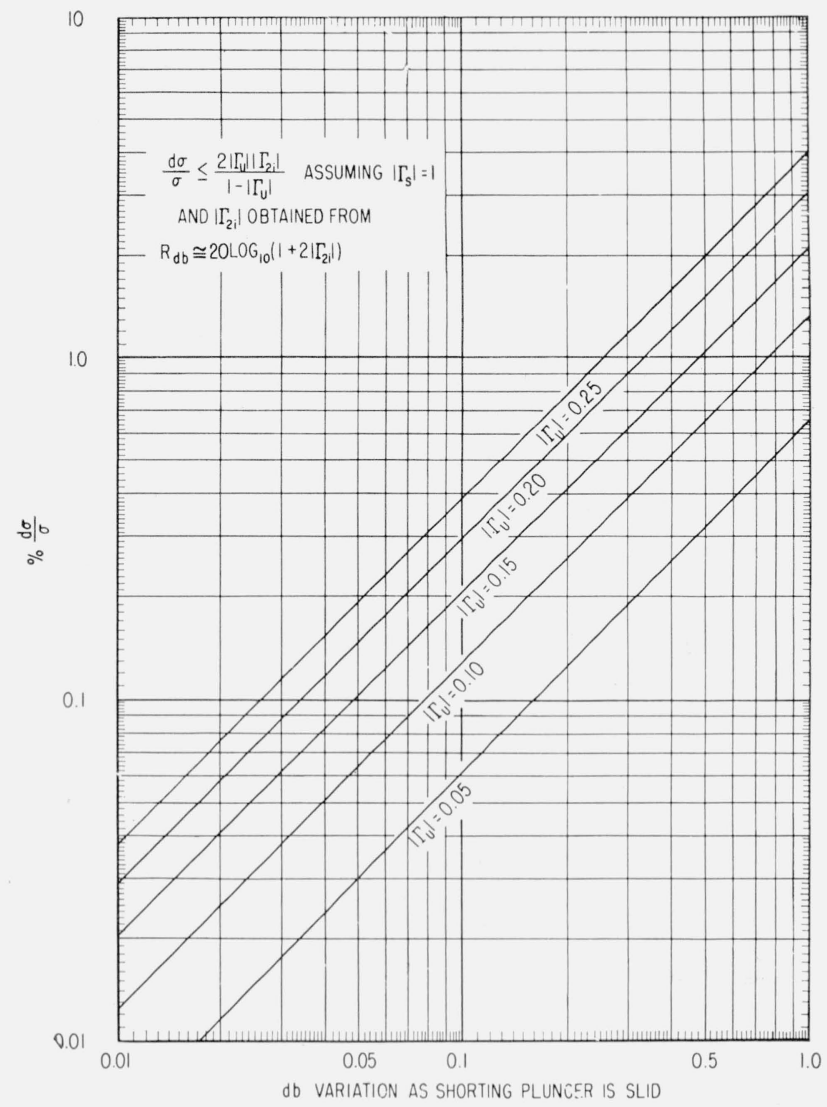

FIgURE 6. The maximum error in measuring VSWR by the modified reflectometer technique due to $\Gamma_{2 i} \neq 0$ and assuming $S_{31}=0$.

through (D) repeatedly will systematically reduce the variation. If the response decreases, i.e., moves away from the average of the variation, adjustment of that stub will probably be useless and another stub should be tried.

The adjustments for no variation as the load is slid are usually quicker if several effective stubs are located by this procedure and then alternately adjusted.

If no effective stubs can be located, make some random adjustment of the tuner to raise or lower the detector response and repeat the previous procedure. This is a trial and error process. Experience with your own equipment will provide "feel" for the detector response level resulting when the sliding load variations have been reduced.

2. An approximation to the detector response level resulting when the variation in the sidearm output has been reduced for the infinite directivity can be obtained as follows:

A. Slide the low reflection termination for a minimum.

B. Adjust tuner A for a null.

C. Slide the low reflection termination for a maximum. About $6 \mathrm{db}$ down from the maximum is the detector response level resulting when infinite directivity is achieved. This level can serve as a guide when making the adjustments for no variation as the load is slid. 


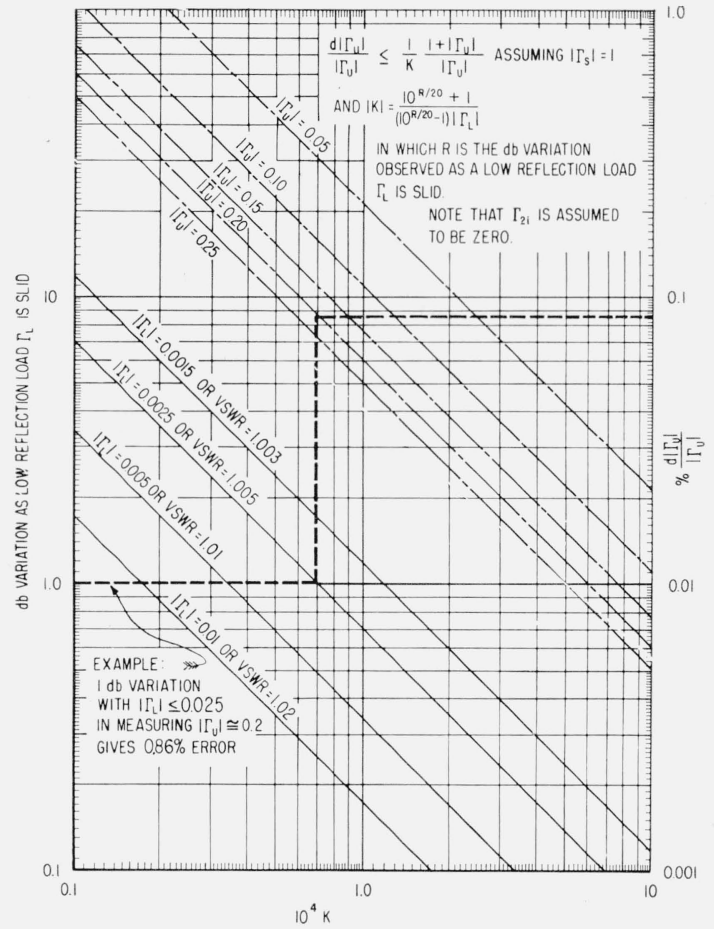

Figure 7. The maximum error in measuring $\left|\Gamma_{u}\right|$ by the modified reflectometer technique due to $S_{31} \neq 0$ and assuming $\Gamma_{2 i}=0$.

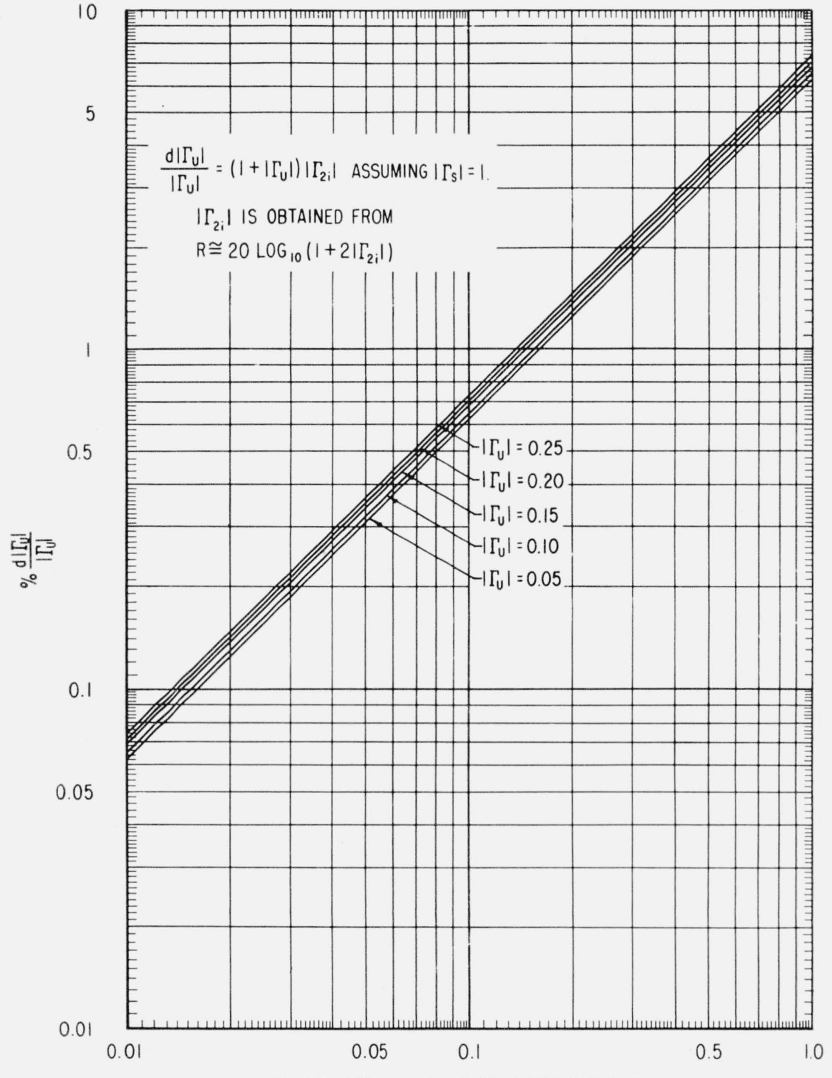

db VARIATION AS SHORTING PLUNGER IS SLID

ligure 8. The maximum error in measuring $\left|\Gamma_{u}\right|$ by the modified reflectometer technique due to $\Gamma_{2 i} \neq 0$ and assuming $\mathrm{S}_{31}=0$.
This procedure avoids the one possible false adjustment which could result in an approximately constant output with $\quad\left|\frac{1}{K}\right| \gg\left|\Gamma_{T}\right|$. This misleading adjustment is seldom encountered except when adjusting the equipment the first time. It sometimes occurs at that time because the system lacks sufficient power sensitivity for detection of the approximately constant output signifying $\left|\frac{1}{K}\right|<<\left|\Gamma_{T}\right|$ which occurs at a low power level.

3 . It is recommended that the adjustments for $S_{31}$ and $\Gamma_{2 i}$ be checked both before and after a VSWR or reflection coefficient measurement is made to detect possible changes created by accidental bumps or frequency drift.

4. The reflection coefficient magnitude $\left|\Gamma_{L}\right|$ of the small reflection load need not be determined to any great accuracy. Computation of the maximum error requires only that the actual value of $\left|\Gamma_{L}\right|$ is less than the value used in the computation.

This reflection coefficient magnitude can be determined accurately enough as follows:

A. Make the normal adjustments of the system, repeating the adjustment sequence until adjustment for $\Gamma_{2 i}=0$ does not require further adjustment of $S_{31}=0$.

B. Slide the small reflection load and observe the maximum and minimum $\left|b_{3}\right|$. There should be little, if any, detectable difference between the minimum and the maximum. Therefore, it is usually sufficient to position the sliding load for the average response and measure $\left|\Gamma_{L}\right|$ for this position using the magnified reflectometer technique. For a truly conservative figure, however, measure the reflection coefficient magnitude of the load in the position of minimum $\left|b_{3}\right|$.

If the standard termination being used is a "short" $\left(\left|\Gamma_{s}\right|=1\right)$ the ratio $R=20 \log _{10} \frac{\left|\Gamma_{s}\right|}{\left|\Gamma_{u}\right|}$ may be too large for the calibrated attenuator of the system. Then it may be necessary to use a standard of smaller reflection coefficient in order to measure $\left|\Gamma_{u}\right|$.

5 . The adjustment of the tuners as outlined in 5.1 is an efficient technique if effective stubs can be found. The following technique aids in finding effective stubs for the infinite directivity adjustment.

A. Adjust tuner $\mathrm{A}$ and the sliding load for $\left|b_{3}\right|=0$.

B. Slide the load for maximum $\left|b_{3}\right|$.

C. Now at least one of the stubs is an "effective" stub. Continue the adjustment as outlined in 5.1.

6 . When using the small reflection load $\left(\left|\Gamma_{L}\right|<\right.$ $0.005)$ the signal level may disappear into the noise as the adjustments for infinite directivity are attempted. This difficulty indicates that the detection system does not have sufficient power sensitivity. Kather than invest in more sensitive equipment, a load with greater reflection (perhaps $\left|\Gamma_{L}\right| \sim 0.025$ ) can be used for the infinite directivity adjustment provided the adjustment sequence outlined in section 3.1 is repeated as indicated. However, this alternate procedure is considerably more time consuming. 
The author is indebted to Edward Niesen for his invaluable assistance in the operation and maintenance of the equipment, to R. W. Beatty for his encouragement and helpful suggestions during the writing of this guide, and to David Wait and Michael Brady for their work on the error analysis.

\section{References}

Beatty, R. W., Mismatch errors in the measurement of ultra high frequency and microwave variable attenuators, J. Research NBS 5\%, 7 (Jan. 1954).

Beatty, R. W., An adjustable sliding termination for rectangular waveguide, IRE Trans. on Microwave Theory and Tech. 5, No. 3 (July 1957).

Beatty, R. W., Magnified and squared VSWR responses, IRE Trans. on Microwave Theory and Tech. 7 , No. 3 (July 1959).

Beatty, R. W., and W. J. Anson, Table of magnitude of reflection coefficient versus return $\operatorname{loss}\left(\mathrm{L}_{R}=20 \log _{10} \frac{1}{K}\right)$, NBS Tech. Note 72 (Sept. 1960).
Beatty, R. W., G. F. Engen, and W. J. Anson, Measurement of reflections and losses of waveguide joints and connectors using microwave reflectometer techniques, IRE Trans. on Instrumentation I-9, No. 2, 219-226 (Sept. 1960).

Beatty, R. W., and D. M. Kerns, Recently developed microwave impedance standards and methods of measurement, IRE Trans. on Instrumentation, p. 319 (Dec. 1958).

Engen, G. F., A method of improving isolation in multichannel waveguide systems, IRE Trans. on Microwave Theory and Tech. 8, p. 460 (July 1960).

Engen, Glenn F., and R. W. Beatty, Microwave reflectometer techniques, IRE Trans. on Microwave Theory and Tech. 351-355 (July 1959).

Schafer, G. E., and A. Y. Rumfelt, Mismatch errors in cascade connected variable attenuators, IRE Trans. on Microwave Theory and Tech. $\boldsymbol{\gamma}$, p. 447 (Oct. 1959).

(Paper 65C4-74) 